\title{
Gene expression profiling reveals differential effects of sodium selenite, selenomethionine, and yeast-derived selenium in the mouse
}

\author{
Jamie L. Barger · Tsuyoshi Kayo • Thomas D. Pugh • \\ James A. Vann · Ronan Power · Karl Dawson • \\ Richard Weindruch · Tomas A. Prolla
}

Received: 24 May 2011 / Accepted: 21 July 2011/Published online: 17 August 2011

(C) The Author(s) 2011. This article is published with open access at Springerlink.com

\begin{abstract}
The essential trace mineral selenium is an important determinant of oxidative stress susceptibility, with several studies showing an inverse relationship between selenium intake and cancer. Because different chemical forms of selenium have been reported to have varying bioactivity, there is a need for nutrigenomic studies that can comprehensively assess whether there are divergent effects at the molecular level. We examined the gene expression profiles associated with selenomethionine (SM), sodium selenite (SS), and yeast-derived selenium (YS) in the intestine, gastrocnemius, cerebral cortex, and liver of mice. Weanling mice were fed either a selenium-deficient (SD) diet $(<0.01 \mathrm{mg} / \mathrm{kg}$ diet $)$ or a diet supplemented with one of three selenium sources $(1 \mathrm{mg} / \mathrm{kg}$ diet, as either SM, SS or YS) for 100 days. All forms of selenium were equally effective in activating standard measures of selenium status, including tissue selenium levels, expression of genes encoding selenoproteins (Gpx1 and Txnrd2), and
\end{abstract}

Electronic supplementary material The online version of this article (doi:10.1007/s12263-011-0243-9) contains supplementary material, which is available to authorized users.

J. L. Barger $(\square) \cdot$ T. Kayo · T. D. Pugh · J. A. Vann

LifeGen Technologies, LLC, 510 Charmany Drive Suite 263,

Madison, WI 53719, USA

e-mail: Jamie.L.Barger@gmail.com

R. Power · K. Dawson

Alltech Biotechnology, Nicholasville, KY, USA

R. Weindruch

Department of Medicine and Veterans Administration Hospital, University of Wisconsin-Madison, Madison, WI, USA

T. A. Prolla

Departments of Genetics and Medical Genetics,

University of Wisconsin, Madison, WI, USA increasing GPX1 enzyme activity. However, gene expression profiling revealed that SS and YS were similar (and distinct from SM) in both the expression pattern of individual genes and gene functional categories. Furthermore, only YS significantly reduced the expression of Gadd $45 \mathrm{~b}$ in all four tissues and also reduced GADD45B protein levels in liver. Taken together, these results show that gene expression profiling is a powerful technique capable of elucidating differences in the bioactivity of different forms of selenium.

Keywords Selenium - Genomics · Nutrigenomics · DNA damage

\section{Introduction}

The trace mineral selenium plays a key role in several biological processes, including the response to oxidative stress (Brenneisen et al. 2005), DNA damage and repair (Seo et al. 2002a, b), cancer susceptibility (Rayman 2005), and viral pathogenicity (Beck 2007). Selenium is fed to animals and humans either as inorganic salts, such as sodium selenite (SS) and selenate, as selenomethionine (SM) or as yeast-derived selenium (YS) that contains selenium as protein-bound SM and other less characterized selenium organic compounds (McSheehy et al. 2005; Ip et al. 2000). While some studies suggest that inorganic selenium (selenite or selenate) is less bioavailable or less bioactive than SM or YS (Rider et al. 2010; Mahmoud and Edens 2003; Qin et al. 2007), other studies suggest that the source of selenium does not differentially affect parameters such as growth or expression and activity of selenoproteins (Qin et al. 2007, 2009; Wang et al. 2010). A human study that addressed the effects of different forms of selenium on 
selenium-replete subjects suggests that plasma selenium reflects the SM content of yeast and that selenium in the form of SM is better absorbed than SS based on urinary selenium excretion (Burk et al. 2006).

The selection of an appropriate source of selenium for supplementation studies is relevant to human health because extensive epidemiological data suggest a link between selenium status and cancer at various sites, and a number of trials testing the effects of selenium supplementation in cancer chemoprevention have led to positive results (Reid et al. 2006; Duffield-Lillico et al. 2003). One study in humans that involved selenium supplementation in the form of YS was associated with a marked reduction in cancer incidence and mortality (Clark et al. 1996). These and other observations led to the design of the SELECT study, a phase III randomized, placebo-controlled trial testing the role of SM and/or vitamin E supplementation on prostate cancer incidence. The trial was terminated early due to observations suggesting negative effects of selenium and/or vitamin E intake. However, a major concern in the design of this study was the selection of SM as the chemical form of selenium to be used; SM was selected as it is the most abundant selenium chemical form in YS, and also because the chemical composition of independent batches of YS was thought to be variable (Lippman et al. 2005, 2009). Nevertheless, the use of SM in a study designed to confirm a previous study performed with a different chemical form of selenium seems problematic and could yield contradictory results. In the absence of detailed knowledge of the biological properties of different selenium chemical forms, rational choice of chemical source of selenium for chemoprevention studies is not possible.

Because mechanistic studies of different forms of selenium at the molecular level are lacking, we investigated the effects of SS, SM, and YS on several parameters in the mouse, including global gene expression profiles in multiple tissues, the effects on key selenoproteins, and oxidative DNA damage. Our findings suggest striking differences regarding the biological activities of different chemical forms of selenium.

\section{Materials and methods}

Animals and diets

Male and female C57BL6/J mice were purchased from Jackson Labs (Bar Harbor, Maine), maintained as breeding pairs, and received LabDiet 5001 ad libitum. Immediately after weaning at 21 days, male mice were randomly assigned to $a$ selenium-deficient diet (SD) or a diet containing $1 \mathrm{mg}$ selenium $/ \mathrm{kg}$ diet from one of three sources: L-selenomethionine (SM, Sigma-Aldrich, St. Louis,
Missouri), sodium selenite (SS, Sigma-Aldrich, St. Louis, Missouri), or yeast selenium (YS, Sel-Plex ${ }^{\circledR}$, Alltech Inc., Nicholasville, Kentucky).

Experimental diets were torula yeast-based diets, prepared by Harlan-Teklad (Madison, WI) and described in detail elsewhere (Rao et al. 2001). We supplemented the SD, SS, and SM diets with an equal amount of non-seleniumenriched yeast (selenium $<0.5 \mathrm{ppm}$ on a product basis) to control for the effects of non-selenium-related yeast components. Selenium levels in dietary premixes were evaluated by atomic absorption spectroscopy (Connolly et al. 2003); selenium level in the SD diet was confirmed to be $<0.03 \mathrm{ppm}$, whereas levels in the supplemented diets were $\mathrm{YS}=1.05 \mathrm{ppm}, \mathrm{SS}=0.99 \mathrm{ppm}$, and $\mathrm{SM}=1.02 \mathrm{ppm}$. Mice were housed two to three per cage, and food and water were provided ad libitum. At 100 days of age, mice were euthanized by cervical dislocation, and tissues were rapidly dissected, flash-frozen in liquid nitrogen, and stored at $-80^{\circ} \mathrm{C}$ for later analysis. All procedures were approved by the Animal Care Committee at the William S. Middleton Veterans Administration Hospital.

\section{Selenium analysis in tissues}

We focused our studies on four tissues: cerebral cortex, small intestine (3-cm section corresponding to the jejunum), gastrocnemius muscle, and liver. Tissues from seven mice per diet were used for measurement of selenium content using molecular fluorescence spectrometry following wet digestion and reaction with 2,3-diaminonapthalene [described previously (Koh and Benson 1983)]. Bovine liver Standard Reference Material from the National Institute of Standards and Technology was used as a standard. Dietary effects were analyzed by one-way analysis of variance; if the overall treatment effect was statistically significant $(P<0.05)$, differences between individual diets were determined using Tukey's post hoc tests.

Gene expression profiling and pathway analysis

For each of the four tissues described above, we performed gene expression profiling on five mice from each diet. Affymetrix Mouse Genome 430A arrays were used to measure gene expression in the intestine, and Mouse Genome 4302.0 arrays were used for gastrocnemius muscle, cerebral cortex, and liver. At the time of the analysis, the Mouse Genome 430A array represented 12,445 unique genes, and the Mouse Genome 4302.0 array represented 20,318 unique genes. Details regarding sample preparation and array hybridization are described elsewhere (Lee et al. 1999). Briefly, total RNA was isolated from individual tissues using TRIzol (Life Technologies) and was processed to biotin-labeled cRNA according to 
Affymetrix protocols. Microarrays were scanned with the Affymetrix GeneArray Scanner (Affymetrix), and the value for each RNA abundance was automatically calculated with the Affymetrix GeneChip Analysis Suite version 3.3 after scanning. When a gene was represented by multiple probe sets on an array, only the probe set having the greatest signal intensity (averaged across all 20 arrays within a tissue) was included for analysis. A gene was considered to be significantly changed in expression when the $P$ value for a two-tailed $t$-test was $<0.01$.

We performed a pathway analysis using parametric analysis of gene set enrichment (PAGE) to identify gene functional classes that were affected by selenium supplementation (Kim and Volsky 2005). Gene expression data were annotated with functional data from the Gene Ontology (GO) consortium (http://www.geneontology.org). We only analyzed GO terms that were annotated at Level 3 or greater and were represented by at least 10 but not more than 1,000 genes. A GO term was considered to be significantly changed by treatment if the $P$ value was $<0.01$.

\section{Real-time RT-PCR confirmation of DNA}

microarray results

To confirm the DNA microarray findings, we used gastrocnemius muscle and liver from the microarray study to measure the expression of two genes changed by all diets using reverse transcriptase quantitative PCR (RT-qPCR; glutathione peroxidase $1, \mathrm{Gpx} 1$, and thioredoxin reductase 2, Txnrd2). The RT-qPCR assay was performed using primers from Applied Biosystems on an Eppendorf realplex ${ }^{2}$ instrument using the $\delta-\delta \mathrm{C}_{\mathrm{t}}$ method as described previously (Barger et al. 2008a). Beta-2-microglobulin (B2m) and mitochondrial ribosomal protein L13 (Mrpl13) were used as normalizing genes in liver and muscle, respectively, because the microarray data revealed that they were abundantly expressed and unchanged by any selenium treatment. Dietary effects were analyzed by one-way analysis of variance; if the overall treatment effect was statistically significant $(P<0.05)$, differences between individual diets were determined using Tukey's post hoc tests.

Western blot analysis and selenoprotein activity

Microarray analysis also revealed that the growth arrest and DNA-damage-inducible 45 beta gene (Gadd45b) was decreased in expression by one or more selenium sources in every tissue studied. To confirm this finding at the protein level, we quantified the abundance of the protein encoded by this gene by western blotting in liver. Tissues were homogenized on ice in seven volumes of cold protein extraction buffer that consisted of $20 \mathrm{mM}$ HEPES pH 7.9, $125 \mathrm{mM} \mathrm{NaCl}, 0.1 \%$ Igepal (NP-40), 0.1\% Triton X-100, and $1 \mathrm{mM}$ EDTA. Homogenates were clarified by centrifugation at $4^{\circ} \mathrm{C}$ for $10 \mathrm{~min}$ at $18,000 \mathrm{~g}$. Supernatants were aliquoted and stored at $-80^{\circ} \mathrm{C}$. Samples for western blotting were electrophoresed in NuPAGE $7 \%$ polyacrylamide Tris-acetate gels (Invitrogen) and transferred to nitrocellulose membranes using the iBlot Dry Blotting System (Invitrogen). Membranes were blocked in $0.5 \%$ gelatin in TBST (137 mM NaCl, 0.1\% Tween-20, 20 mM Tris, pH 7.6) for $1 \mathrm{~h}$. Primary and secondary antibodies were diluted in $0.5 \%$ gelatin in TBST. Rabbit polyclonal antiGADD $45 \beta$ (H-70) was purchased from Santa Cruz Biotechnology (Santa Cruz, CA). HRP-linked goat anti-rabbit IgG was purchased from Pierce (Rockford, IL) as part of the Dura SuperSignal West chemiluminescent substrate kit, which was used to detect antibodies. Chemiluminescent bands were visualized and analyzed using a UVP Bioimaging Systems (Upland, CA). MemCode ${ }^{\mathrm{TM}}$ Reversible Protein Stain Kit was purchased from Pierce and used to control for protein loading. A loading correction factor based on the memcode protein band intensity data was used to adjust the GADD $45 \beta$ band intensity data.

Glutathione peroxidase and thioredoxin reductase enzyme activities in liver were measured using commercially available kits (Cayman Chemical); protein homogenates were prepared from the same source material used in the microarray analysis, and enzyme activity was measured according to the manufacturer's instructions. Dietary effects were analyzed by one-way analysis of variance; if the overall treatment effect was statistically significant $(P<0.05)$, differences between individual diets were determined using Tukey's post hoc tests.

\section{Oxidative damage to DNA}

DNA damage was quantified from DNA isolated from liver tissue of mice using a highly sensitive HPLC/EC/UV system as described previously (Barger et al. 2008b). Samples were compared against a calibration curve with known standards to quantify the levels of the oxidative products 8-hydroxy- $2^{\prime}$ deoxyguanosine $/ 10^{6} 2^{\prime}$-deoxyguanosine (8-oxo-dG). Data were analyzed by one-way analysis of variance; if the overall treatment effect was statistically significant $(P<0.05)$, differences between individual diets were determined using Tukey's post hoc tests.

\section{Results}

Effects of experimental diets on tissue selenium

Mice consuming the SD diets had low selenium levels in all tissues examined, below $0.1 \mu \mathrm{g}$ selenium/g tissue (Fig. 1). When compared to the SD diet, SM and YS 
significantly elevated selenium levels in all four tissues; SS elevated tissue selenium levels compared to SD in all tissues except for gastrocnemius. When comparing the different sources of selenium to one another, the effect of diet was different depending on the tissue studied: In cerebral cortex and intestine, levels of selenium were highest in SM, intermediate in YS, and lowest in the SS diet (SM > YS > SS); in gastrocnemius, selenium levels were similar between SM and YS diets, but higher than the SS $\operatorname{diet}(\mathrm{SM}=\mathrm{YS}>\mathrm{SS})$. In liver, selenium levels were highest in the SM, intermediate in the YS, and lowest in the $\mathrm{SS} \operatorname{diet}(\mathrm{SM} \geq \mathrm{YS} \geq \mathrm{SS})$.

\section{Overview of gene expression patterns}

Selenium supplementation with SM, SS, or YS resulted in the differential expression of many (hundreds to thousands) of genes, with some tissues being more responsive than others (Fig. 2). Gastrocnemius and cerebral cortex were the most responsive to selenium supplementation, with 21 and $17 \%$, respectively, of the genes represented on the array being changed in expression by at least one diet. In liver, $12 \%$ of the genes represented on the array were changed in expression by at least one diet, and in the intestine, only $4 \%$ of the genes represented on the array were changed in expression by at least one diet. Despite the fact that tissue

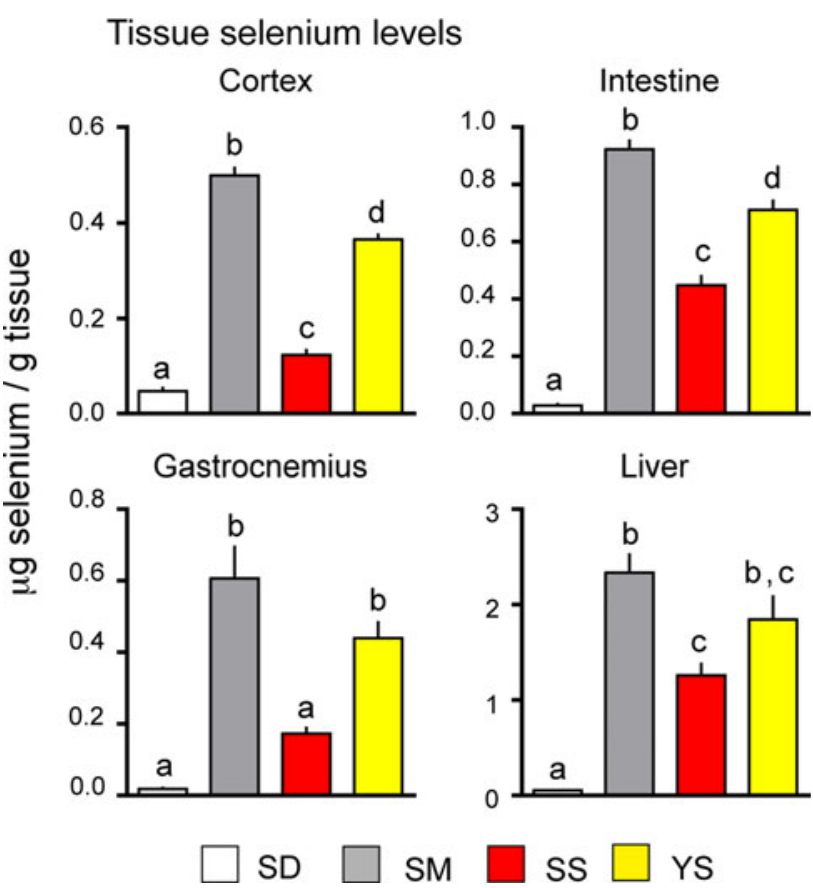

Fig. 1 Tissue levels of selenium (mean + SEM) in four tissues from mice fed a selenium-deficient $(S D)$ or selenium-supplemented diet ( $S M$ selenomethionine, $S S$ sodium selenite, and $Y S$ yeast selenium). Within a tissue, different letters indicate a statistically significant difference between treatments $(P<0.05)$ selenium levels were highest in response to the SM diet, SM supplementation affected the expression of the least number of genes (compared to SS and YS). In contrast, tissue selenium levels were lowest in response to the SS diet, but SS affected the expression of more genes than either SM or YS.

For each tissue, there were groups of genes changed in expression by only one selenium source, groups of genes changed by two selenium sources, and a group of genes changed in expression by all three treatments (Fig. 2). In general, if a gene was significantly changed in expression by more than one selenium source, the direction of the change in expression (increased or decreased) was similar for the other treatment(s); this similarity is represented by the fractions within the intersecting regions of Fig. 2. A remarkable finding was that the overlap between SS and YS was higher than that of any other pair of treatments. For example, in the small intestine, 86 genes were significantly changed in expression by both the SS and YS diets,
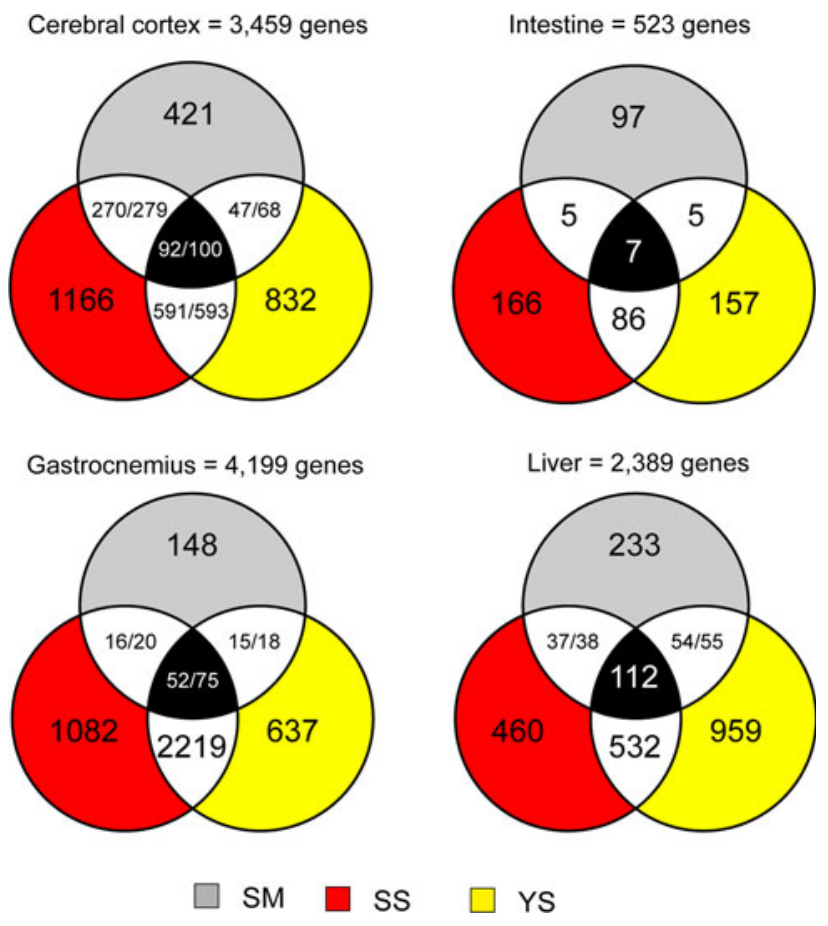

Fig. 2 Effect of selenium supplementation on the number of differentially expressed $(P<0.01)$ genes in four tissues of mice. When genes were changed by more than one treatment (shown in intersecting regions), the denominator indicates the total number of genes changed and the numerator indicates how many of those genes were changed in the same direction by all treatments. If a ratio is not shown for a given intersection, all genes were changed in the same direction by all treatments. As described in the "Materials and methods," there were fewer genes represented on the array used for the intestine which explains, in part, the lower number of differentially expressed genes in this tissue; the percentage of differentially expressed genes (relative to the total number of genes represented on the array) for each tissue is stated in the "Results" 
whereas only five genes were significantly changed by both SM and SS diets and SM and SY diets (an abbreviated list of genes changed in expression by more than one diet in small intestine is shown in Table 1). This large similarity between SS and YS diets was also observed in the other three tissues as shown in the overlap in the Venn diagrams in Fig. 2 (indicated by the greatest number of genes in the overlap between SS and YS circles). A complete list of the individual genes changed in expression by more than one diet for all four tissues is shown in Online Resources 1-4. Thus, the gene expression profile of YS is much more similar to that of SS, despite the fact that SM is a major component of YS. Perhaps surprisingly, there was no gene that was significantly changed in expression at $P<0.01$ in all tissues by all selenium diets. However, selenoprotein $\mathrm{W}$ was increased in expression in all tissues and diets as compared to $\mathrm{SD}$ at a lower level of statistical significance ( $P<0.05$, data not shown). As discussed below, Gadd45b was the only gene not related to selenium biology that was changed in expression by multiple diets in every tissue.

Using RT-PCR, we examined the expression of seven genes that showed diet-specific changes in expression in the microarray analysis (Table 2). Three of these genes (Cyp7b1, Sucnr1, and Tsc22d1) showed an identical pattern of gene expression for both the microarray and RT-PCR analyses. For the remaining genes, the direction of the fold change in expression was similar between the two techniques, but the number of significantly different genes varied.

\section{Pathway analysis}

Similar to what was observed in the analysis of individual genes (above), pathway analysis revealed that different selenium sources had both individual and overlapping effects on functional classes of genes in each tissue (Online Resource 5-8). Figure 3 represents GO terms changed by at least $2 / 3$ diets and shows that all three diets tended to have similar effects on both gastrocnemius and liver (Fig. 3a, c, respectively), with SM having a less pronounced effect in gastrocnemius. In the intestine and cerebral cortex, however, the overall effect of the SM diet tended to be the opposite of both the SS and YS diets (Fig. 3b, d, respectively).

Because GO term "mitochondrial inner membrane" (GO:0005743) was significantly regulated by at least two treatments in each tissue (Fig. 3a-d), this pathway provides a useful parameter for comparison among the different selenium sources. In gastrocnemius, the class of genes representing the mitochondrial inner membrane was upregulated by all diets; in the liver, this gene class was upregulated by SM and YS; in the intestine, this gene class was upregulated by SM but downregulated by both SS and YS. In cerebral cortex, SM and YS downregulated this class of genes, but SS upregulated this gene class overall. Thus, different sources of dietary selenium clearly have different effects at the gene expression level.

Analysis of selenoproteins

The functional category that was most consistently and robustly modulated by all selenium diets was the GO term "selenium binding" (GO:0008430), which is defined as a class of genes that "interact selectively and non-covalently with selenium." This GO term was significantly upregulated by all three diets in every tissue except for cortex (Fig. 4a). The expression of two key genes in this GO term was confirmed by RT-qPCR in liver: Gpx 1 and Txnrd2 expression was increased approximately five- and twofold in expression, respectively, by all three diets relative to the SD group (Fig. 4b). Total glutathione peroxidase enzymatic activity in liver was also significantly increased nearly eightfold by all three selenium diets, whereas thioredoxin reductase activity in liver was significantly increased threefold by the SM diet, with an intermediate level of activity in the SS and YS diets (Fig. 4c).

\section{Gadd45b and DNA oxidation}

In a search for genes unrelated to selenium biology that were consistently regulated by selenium supplementation across tissues, we observed that the gene Gadd45b was decreased in expression by all selenium diets in cerebral cortex, decreased by SS and YS in gastrocnemius, and decreased by YS only in intestine and liver (Fig. 5a). Gadd45b is thought to be an important mediator of the DNA damage response, and its expression is induced with DNA damage or aging (Liu et al. 2009; Hoffman and Liebermann 2009). Because this gene was decreased in expression by selenomethionine in a previous study (Rao et al. 2001), we expanded upon the microarray findings by measuring the abundance of the protein encoded by this gene. GADD45B protein was not different in SM and SS diets compared to the SD diet, but was significantly reduced by $44 \%$ in liver of the YS diet (Fig. 5b). Finally, we measured levels of DNA oxidation (8-oxo-dG) in the liver of mice fed the four diets. The amount of oxidized DNA was not significantly affected by selenium supplementation, though there was a trend toward a decreased level of oxidized DNA (27\%) in the YS diet (Fig. 5c).

\section{Discussion}

We used a nutrigenomic approach to assess the effects of three different forms of selenium in four tissues from mice. Because all forms of selenium corrected the alterations in 
Table 1 Selected microarray data from intestine for genes changed in expression by at least two diets $(P<0.01)$
See Online Resource 3 for complete list

\begin{tabular}{|c|c|c|c|c|}
\hline Gene symbol & Gene name & $\begin{array}{l}\mathrm{FC} \\
(\mathrm{SM})\end{array}$ & $\begin{array}{l}\mathrm{FC} \\
(\mathrm{SS})\end{array}$ & $\begin{array}{l}\mathrm{FC} \\
(\mathrm{YS})\end{array}$ \\
\hline Fasl & Fas ligand (TNF superfamily, member 6) & 3.63 & 5.35 & nsd \\
\hline Dolpp1 & Dolichyl pyrophosphate phosphatase 1 & 1.37 & 1.46 & nsd \\
\hline Srsf2ip & Serine/arginine-rich splicing factor 2 , interacting protein & -1.73 & -1.82 & nsd \\
\hline Pla1a & Phospholipase A1 member A & 1.84 & nsd & 2.26 \\
\hline Txnrd1 & Thioredoxin reductase 1 & 1.7 & nsd & 1.86 \\
\hline Odc1 & Ornithine decarboxylase, structural 1 & 1.44 & nsd & 1.64 \\
\hline Aadat & Aminoadipate aminotransferase & -2.84 & nsd & -2.91 \\
\hline Lrp5 & Low-density lipoprotein receptor-related protein 5 & nsd & 4.25 & 3.92 \\
\hline Dmpk & Dystrophia myotonica-protein kinase & nsd & 2.99 & 3.32 \\
\hline Anpep & Alanyl (membrane) aminopeptidase & nsd & 2.45 & 2.62 \\
\hline Polr2a & Polymerase (RNA) II (DNA directed) polypeptide A & nsd & 2.09 & 2.52 \\
\hline Rasa2 & RAS p 21 protein activator 2 & nsd & 2.65 & 2.33 \\
\hline Scly & Selenocysteine lyase & nsd & 1.89 & 2.33 \\
\hline Leng8 & Leukocyte receptor cluster (LRC) member 8 & nsd & 2.22 & 2.29 \\
\hline Oxct1 & 3-Oxoacid CoA transferase 1 & nsd & 2.24 & 2.23 \\
\hline Dak & Dihydroxyacetone kinase 2 homolog (yeast) & nsd & 1.83 & 2.23 \\
\hline 2210023G05Rik & RIKEN cDNA 2210023G05 gene & nsd & 2.52 & 2.2 \\
\hline Syvn1 & Synovial apoptosis inhibitor 1 , synoviolin & nsd & 1.93 & 2.18 \\
\hline Ncstn & Nicastrin & nsd & 1.9 & 2.18 \\
\hline Brwd1 & Bromodomain and WD repeat domain containing 1 & nsd & 2.38 & 2.1 \\
\hline Fmo5 & Flavin containing monooxygenase 5 & nsd & 2.27 & 2.06 \\
\hline $\mathrm{Cic}$ & Capicua homolog (Drosophila) & nsd & 2.06 & 2.05 \\
\hline Tra2a & Transformer 2 alpha homolog (Drosophila) & nsd & 2.21 & 2.04 \\
\hline Mical2 & $\begin{array}{l}\text { Microtubule associated monoxygenase, calponin and LIM } \\
\text { domain containing }\end{array}$ & nsd & 1.98 & 2.03 \\
\hline Src & Rous sarcoma oncogene & nsd & 2.01 & 2 \\
\hline Gbf1 & Golgi-specific brefeldin A-resistance factor 1 & nsd & 1.98 & 2 \\
\hline Slc30a9 & Solute carrier family 30 (zinc transporter), member 9 & nsd & -1.66 & -1.75 \\
\hline Tprkb & Tp53rk-binding protein & nsd & -1.5 & -1.79 \\
\hline Ppp1cb & Protein phosphatase 1, catalytic subunit, beta isoform & nsd & -1.9 & -1.82 \\
\hline Fzd4 & Frizzled homolog 4 (Drosophila) & nsd & -1.99 & -1.89 \\
\hline Nans & $\mathrm{N}$-acetylneuraminic acid synthase (sialic acid synthase) & nsd & -1.99 & -1.97 \\
\hline Cpd & Carboxypeptidase D & nsd & -2.02 & -2.04 \\
\hline Homer2 & Homer homolog 2 (Drosophila) & nsd & -1.95 & -2.25 \\
\hline Ndufab1 & $\begin{array}{l}\text { NADH dehydrogenase (ubiquinone) } 1 \text {, alpha/beta } \\
\text { subcomplex, } 1\end{array}$ & nsd & -2.45 & -3.07 \\
\hline Sepw1 & Selenoprotein $\mathrm{W}$, muscle 1 & 4.86 & 8.15 & 7.15 \\
\hline 2700094K13Rik & RIKEN cDNA 2700094K13 gene & 5.25 & 3.82 & 4.73 \\
\hline Gpx 1 & Glutathione peroxidase 1 & 4.34 & 4.06 & 4.69 \\
\hline Gpx3 & Glutathione peroxidase 3 & 3.63 & 2.74 & 2.95 \\
\hline Dio1 & Deiodinase, iodothyronine, type I & 1.95 & 2.87 & 2.28 \\
\hline Pias1 & Protein inhibitor of activated STAT 1 & 1.27 & 1.61 & 1.63 \\
\hline Pnpo & Pyridoxine $5^{\prime}$-phosphate oxidase & 1.25 & 1.2 & 1.31 \\
\hline
\end{tabular}

expression of genes encoding selenium-binding proteins and also corrected the reduced glutathione peroxidase activity in the SD diet, we conclude that the different forms of selenium (fed a $1 \mathrm{ppm}$ of the diet) are equivalent in their ability to correct a selenium deficiency. Nonetheless, there appear to be distinct effects of the different forms of selenium: We observed that yeast-derived selenium (YS) results in overall gene expression profiles that are similar to those of SS, despite the fact that YS is thought to contain selenomethionine as the major selenium source (McSheehy 
Table 2 Comparison between microarray and RT-PCR analyses for selected genes

\begin{tabular}{|c|c|c|c|c|c|c|c|}
\hline \multirow[t]{2}{*}{ Gene } & \multirow[t]{2}{*}{ Tissue } & \multicolumn{3}{|c|}{ Microarray fold change ( $P$ value) } & \multicolumn{3}{|c|}{ RT-PCR fold change ( $P$ value) } \\
\hline & & SM & SS & YS & SM & SS & YS \\
\hline Gadd45b & Gastrocnemius & $1.02(0.934)$ & $-2.42(0.011)$ & $-2.16(0.011)$ & $-1.16(0.407)$ & $-1.48(0.183)$ & $-1.87(0.039)$ \\
\hline Crkl & Gastrocnemius & $-1.02(0.801)$ & $-1.29(0.002)$ & $-1.27(0.001)$ & $-1.25(0.000)$ & $-1.30(0.000)$ & $-1.81(0.000)$ \\
\hline Tsc22d1 & Liver & $1.2(0.591)$ & $1.63(0.136)$ & $2.24(0.002)$ & $-1.48(0.136)$ & $1.29(0.271)$ & $2.43(0.030)$ \\
\hline Sucnr1 & Liver & $1.12(0.713)$ & $1.25(0.361)$ & $1.75(0.008)$ & $1.03(0.919)$ & $1.03(0.151)$ & $2.17(0.029)$ \\
\hline Mapre2 & Liver & $-1.30(0.258)$ & $-3.10(0.002)$ & $-3.71(0.001)$ & $-1.33(0.001)$ & $-1.33(0.001)$ & $-1.84(0.001)$ \\
\hline Cyp7b1 & Liver & $1.12(0.758)$ & $1.62(0.181)$ & $1.47(0.002)$ & $1.24(0.701)$ & $1.61(0.201)$ & $1.60(0.009)$ \\
\hline $\mathrm{Nfe} 212$ & Liver & $1.00(0.986)$ & $1.48(0.007)$ & $1.59(0.002)$ & $-1.28(0.081)$ & $1.30(0.357)$ & $1.43(0.027)$ \\
\hline
\end{tabular}

et al. 2005; Ip et al. 2000). In addition, pathway analysis suggests that SM, SS, and YS differentially impact key cellular functions, including mitochondrial function and metabolism. Finally, we found that only YS is associated with a pattern of decreased DNA damage.

We note that all selenium sources resulted in a large number of significant changes in gene expression, ranging from 4 to $21 \%$ of all genes represented in the array for intestine and gastrocnemius, respectively. This finding is in agreement with our previous study of the effects of selenium in mouse intestine, which showed changes in the expression of a large number of genes in response to different selenium sources (Rao et al. 2001). Given the central role of selenium in the cellular antioxidant system, it appears likely that most changes in gene expression in response to alterations in selenium status are secondary to alterations in redox status. Indeed, many transcriptional factors are redox-regulated, including NF-kB (Kabe et al. 2005), NRF2 (Giudice et al. 2010), and the FOXO family of transcriptional factors (Keizer et al. 2011). As an example of such effects, we note that the seleniumdependent thioredoxin reductase modulates thioredoxin activity, which directly regulates the activity of NF-kB and AP-1 (Hirota et al. 1997). Thus, changes in the activity of multiple redox-sensitive transcription factors may lead to alterations in the expression of a large number of genes in response to changes in selenium status.

The observation that gene expression patterns and gene functional categories are highly similar between SS and YS diets was surprising, given that selenomethionine is thought to be the principal selenium form in yeast-derived selenium (McSheehy et al. 2005; Ip et al. 2000). We note that we observed similar effects of SM on the mouse intestine as previously reported by Kipp et al. (2009), including increases in the expression of pathways linked to translation, ribosomal proteins, and RNA processing. One explanation for the differential effects at the gene expression level between selenomethionine and YS in our study is that most selenomethionine found in YS is bound to proteins, whereas selenomethionine in our study was provided as the free amino acid. Differences in metabolism of free and protein-bound selenomethionine may thus account for some of the differences that we observed. A second explanation is that other as yet uncharacterized selenium compounds present in YS account for the differential effects. Yeast-derived selenium contains several organic selenium compounds in addition to SM, as well as the putative cancer chemopreventive compound Se-methyl-Secysteine ( $\left.\mathrm{CH}_{3} \mathrm{SeCys}\right)$ (McSheehy et al. 2005; Ip et al. 2000, 2002). Yet, another possibility is that free selenomethionine is more readily oxidized than methionine, forming a selenoxide as the reaction product (Zainal et al. 1998). Therefore, dietary SM as a free amino acid may not absorbed in this form but instead converted to a biologically less active derivative. Dietary SM can be incorporated nonspecifically into proteins or trans-selenated into SeCys and subsequently $\mathrm{H}_{2} \mathrm{Se}$, a compound that plays a central role in selenium metabolism and serves as a precursor of selenophosphate. Selenophosphate serves as a precursor to selenoprotein synthesis, as well as a precursor to methylselenol, a putative cancer chemopreventive form of selenium (Ip et al. 2002). In contrast, inorganic forms such as SS undergo reductive metabolism, also yielding $\mathrm{H}_{2} \mathrm{Se}$. Because our data suggest striking similarities between SS and YS at the gene expression level, it is possible that similar to SS, the SM found in YS is preferentially metabolized to $\mathrm{H}_{2} \mathrm{Se}$ as compared to free SM. Poor selenium absorption from SM is unlikely to account for our observations, given that SM resulted in high tissue levels of selenium in our study, and recent findings in humans showing higher selenium absorption when provided as SM when compared to YS and SS (Reid et al. 2006).

There is an interest in the relationship among selenium status, DNA damage, and cancer chemoprevention (Rayman 2005). A study that examined individuals in a highrisk group for prostate cancer development identified a significant inverse correlation between DNA damage in leukocytes and serum selenium levels (Waters et al. 2005). Carriers of a BRCA1 mutation are at high risk for breast cancer development, and this mutation is associated with 


\section{A Gastrocnemius}

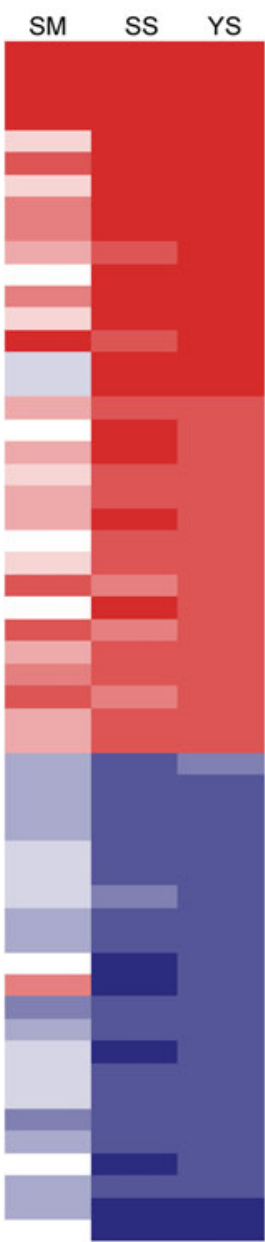

GO:0003735 - structural constituent of ribosome GO:0005840 - ribosome

GO:0006412 - translation

GO:0030529 - ribonucleoprotein complex

GO:0006396 - RNA processing

GO:0033279 - ribosomal subunit

GO:0008380 - RNA splicing

GO:0000313 - organellar ribosome

GO:0005761 - mitochondrial ribosome

GO:0044455 - mitochondrial membrane part

GO:0006397 - mRNA processing

GO:0015934 - large ribosomal subunit

GO:0016070 - RNA metabolic proces

GO:0008430 - selenium binding

GO:0004298 - threonine-type endopeptidase activity

GO:0070003 - threonine-type peptidase activity

GO:0005746 - mitochondrial respiratory chain

GO:0005839 - proteasome core complex

GO:0048193 - Golgi vesicle transport

GO:0022900 - electron transport chain

GO:0044445 - cytosolic part

GO:0006888 - ER to Golgi vesicle-mediated transport

GO:0003723 - RNA binding

GO:0042254 - ribosome biogenesis

GO:0044429 - mitochondrial part

GO:0000502 - proteasome complex

GO:0019866 - organelle inner membrane

GO:0022613 - ribonucleoprotein complex biogenesis and assembly

GO:0019843 - rRNA binding

GO:0005743 - mitochondrial inner membrane

GO:0005762 - mitochondrial large ribosomal subunit

GO:0000315 - organellar large ribosomal subunit

GO:0009892 - negative regulation of metabolic process

GO:0030695 - GTPase regulator activity

GO:0009653 - anatomical structure morphogenesis

GO.000563 - anatomical structure morphogenesis

GO:0005083 - small GTPase regulator activity

GO:0031327 - negative regulation of cellular biosynthetic process

GO:0007243 - protein kinase cascade

GO:0009890 - negative regulation of biosynthetic process

GO:0016481 - negative regulation of transcription

GO:0005924 - cell-substrate adherens junction

GO:0042383 - sarcolemma

GO:0051253 - negative regulation of RNA metabolic process

GO:0016564 - transcription repressor activity

GO:0019218 - regulation of steroid metabolic process

GO:0007242 - intracellular signaling cascade

GO:0010629 - negative regulation of gene expression

GO:0045892 - negative regulation of transcription, DNA-dependent

GO:0000122 - negative regulation of transcription from RNA polymerase II promoter

GO:0005925 - focal adhesion

GO:0006357 - regulation of transcription from RNA polymerase II promoter

GO:0003700 - transcription factor activity

GO:0030865 - cortical cytoskeleton organization

\section{B Intestine}

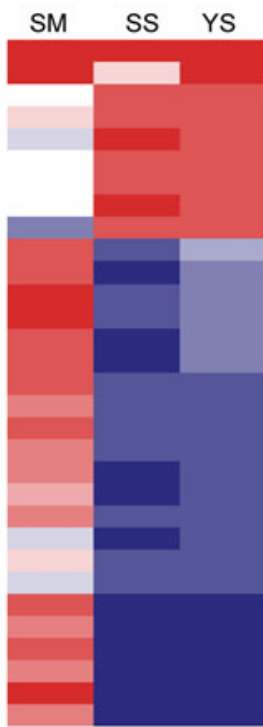

GO:0008430 - selenium binding

GO:0045454 - cell redox homeostasis

GO:0001501 - skeletal system development

GO:0030055 - cell-substrate junction

GO:0009653 - anatomical structure morphogenesis

GO:0005925 - focal adhesion

GO:0005925 - focal adhesion

GO:0005924 - cell-substrate adherens junction

GO:0043687 - post-translational protein modific

GO:0015934 - large ribosomal subunit

GO:0033279 - ribosomal subunit

GO:0033279 - ribosomal subunit

GO:0005759 - mitochondrial matrix

GO:0031980 - mitochondrial lumen

GO:0000313 - organellar ribosome

GO:0005761 - mitochondrial ribosome

GO:0019866 - organelle inner membran

GO:0006399 - tRNA metabolic process

GO:0005743 - mitochondrial inner membrane

GO:0031966 - mitochondrial membrane

GO:0034660 - ncRNA metabolic process

GO:0016070 - RNA metabolic process

GO:0005740 - mitochondrial envelope

GO:0004540 - ribonuclease activity

GO:0004364 - glutathione transferase activity

GO:0006749 - glutathione metabolic process

GO:0044429 - mitochondrial part

GO:0030529 - ribonucleoprotein complex

GO:0005840 - ribosome

GO:0003735 - structural constituent of ribosome

GO:0005739 - mitochondrion

GO:0006412 - translation

\section{Liver}
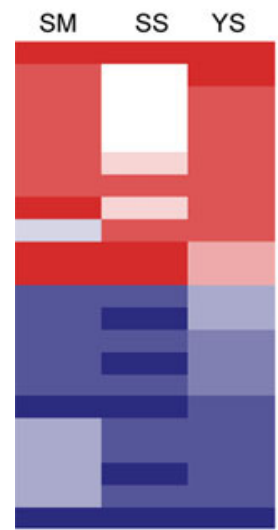

GO:0008430 - selenium binding

GO:0005743 - mitochondrial inner membrane

GO:0031966 - mitochondrial membrane

GO:0005740 - mitochondrial envelope

GO:0019866 - organelle inner membrane

GO:0005746 - mitochondrial respiratory chain

G0.0007519- skeletal muscle development

GO:0008484 - sulfuric ester hydrolase activity

GO:0008484 - sulfuric ester

GO:0003015 - heart process

GO:0006260 - DNA replication

GO:0006260 - DNA replication

GO:0006412 - translation

GO:0006259 - DNA metabolic process

GO:0030529 - ribonucleoprotein comp

GO:0016765 - transferase activity, transferring alkyl or aryl groups

GO:0004298 - threonine-type endopeptidase activity

GO:0070003 - threonine-type peptidase activity

GO:0005839 - proteasome core complex

GO:0006414 - translational elongation

GO:0004364 - glutathione transferase activity

\section{Cerebral cortex}

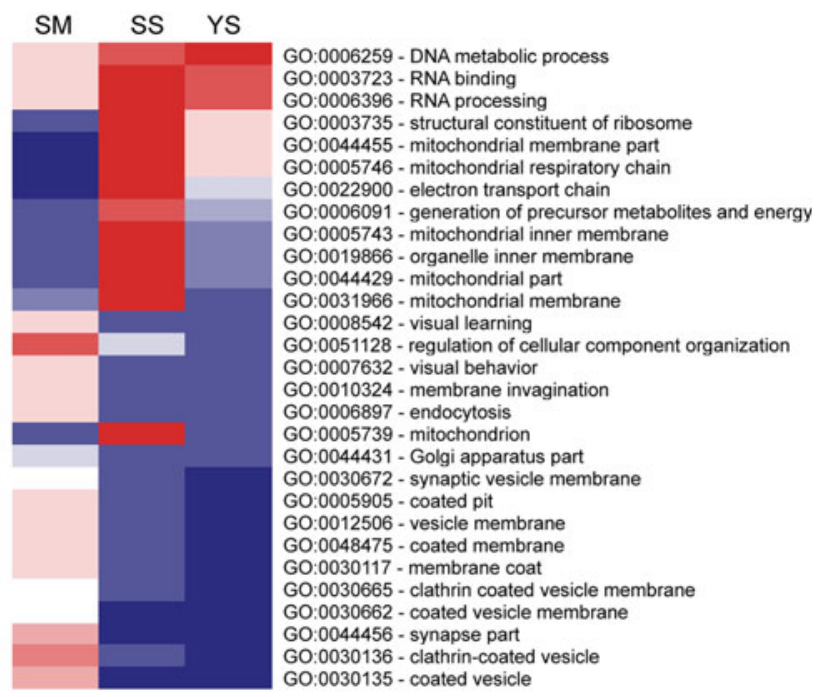

\begin{tabular}{|lllllllllllll}
\hline$>5$ & 4 & 3 & 2 & 1 & $\begin{array}{c}0 \\
\text { z-score }\end{array}$ & -1 & -2 & -3 & -4 & $<-5$
\end{tabular}


Fig. 3 Analysis of Gene Ontology $(G O)$ terms changed by selenium supplementation in four tissues of mice. Terms shown are those significantly changed by at least $2 / 3$ treatments in each tissue $(P<0.001)$. Red indicates a GO term that was upregulated by treatment $(z$-score $>0)$; blue indicates GO terms that were downregulated by treatment $(z$-score $<0)$

increased DNA breakage in response to the oxidant bleomycin (Kowalska et al. 2005). Supplementation with SS normalizes chromosome breakage in this group, supporting a role for selenium in preventing DNA damage or enhancing its repair (Kowalska et al. 2005). However, studies performed in dogs have shown a complex U-shaped relationship between DNA damage in the prostate and selenium levels in response to supplementation (Waters et al. 2003, 2005). We have previously reported, using a

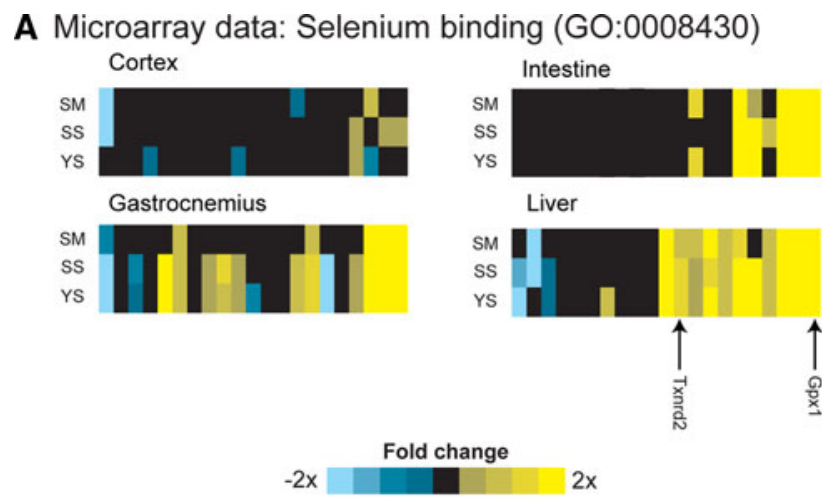

B RT-PCR confirmation of liver microarray data
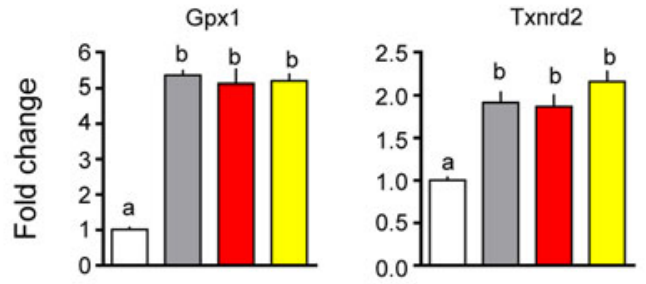

\section{Selenoprotein enzyme activity in liver}

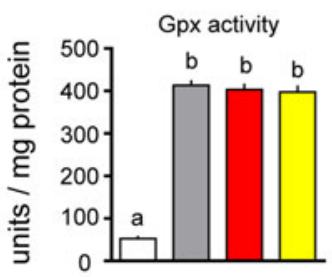

SD

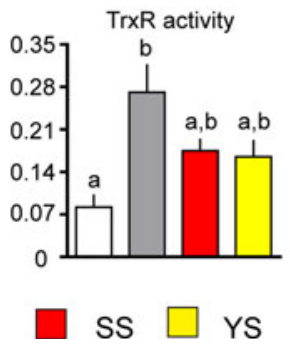

Fig. 4 Effect of selenium supplementation on selenoprotein gene expression and enzyme activity. Values are means \pm SEM; values with different letters indicate statistically significant differences $(P<0.05)$

A Gadd45b expression

Cortex

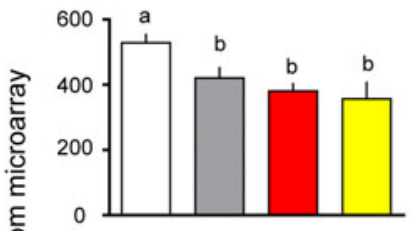

Gastrocnemius

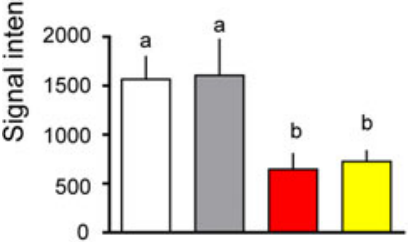

B Liver GADD45B protein

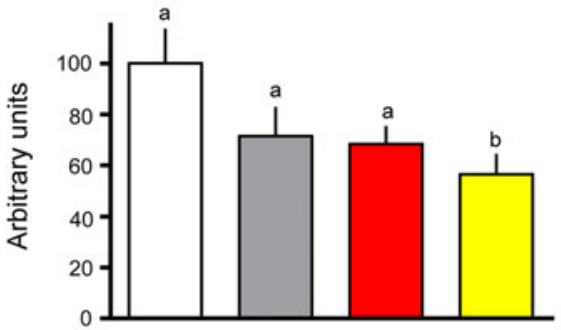

C Liver DNA oxidation

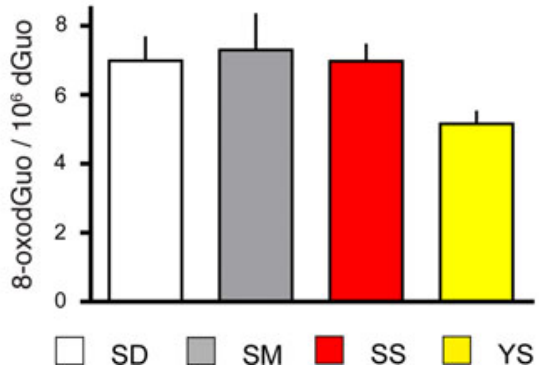

Fig. 5 Effect of selenium supplementation on markers of DNA damage. Values are means $\pm \mathrm{SEM}$; values with different letters indicate statistically significant differences $(P<0.05)$

similar experimental design, that a selenium-deficient diet is associated with the induction of genes linked to DNA damage and oxidative stress in the intestine, including Gadd45b (Rao et al. 2001). Based on these observations, we suggested that the gene expression profile of low selenium status may be associated with tumorigenesis (Rao et al. 2001). We examined the expression of the DNA damage response gene Gadd $45 \mathrm{~b}$ and found that YS consistently lowered its expression in all tissues tested. SS lowered Gadd45b expression in cortex and gastrocnemius, and SM only reduced its expression in cortex. We also observed that in liver, YS significantly reduced the abundance of the protein encoded by the Gadd $45 \mathrm{~b}$ gene. Finally, we measured the levels of a marker of oxidative DNA 
damage (8-oxo-dG) in the liver and found a $27 \%$ reduction in 8-oxo-dG in the YS diet, though this was not statistically significant. These findings are consistent with the recent observation that the same source of YS used in our study significantly reduced both RNA and DNA oxidative damage in the brain in the APP1/PS1 mouse model of Alzheimer's disease (Lovell et al. 2009). Thus, if increased Gadd45b observed with SD reflects chronic cellular stress, in our studies, YS appears to be the most effective form of selenium opposing this stress.

Overall, our study suggests that the different forms of selenium had a similar effect on the expression of seleniumdependent genes and selenoenzyme activity; however, the sources of selenium had differential effects on the overall gene expression patterns (as noted by the similarity between SS and YS) as well as on specific functional pathways related to mitochondrial structure and function. Importantly, we observed that YS alone was associated with an enhanced protection against DNA damage. We note that the SELECT cancer chemoprevention trial was designed partly in response to the previous findings of the Nutrition Prevention of Cancer (NPC) trial, which showed a $63 \%$ reduction in prostate cancer in individuals receiving $200 \mu \mathrm{g}$ /day of yeastderived selenium (Lippman et al. 2005). The divergent gene expression profiles of SM, SS, and YS revealed in this study clearly support the notion of non-equivalency for various chemical forms of selenium and raise the possibility that the choice of selenium source had an impact on the conflicting results of the NPC and SELECT trials. We suggest that the published data regarding the effect of selenium should be re-evaluated with respect to the source of selenium that was administered.

Acknowledgments The authors wish to thank Barb Mickelson for assistance in the formulation of test diets, Kristi Papez for assistance with animal care, Cathal Connolly for analysis of dietary selenium levels, and Don Mahan for analyzing tissue selenium levels.

Open Access This article is distributed under the terms of the Creative Commons Attribution Noncommercial License which permits any noncommercial use, distribution, and reproduction in any medium, provided the original author(s) and source are credited.

\section{References}

Barger JL, Kayo T, Pugh TD, Prolla TA, Weindruch R (2008a) Shortterm consumption of a resveratrol-containing nutraceutical mixture mimics gene expression of long-term caloric restriction in mouse heart. Exp Gerontol 43(9):859-866

Barger JL, Kayo T, Vann JM, Arias EB, Wang J, Hacker TA, Wang Y, Raederstorff D, Morrow JD, Leeuwenburgh C, Allison DB, Saupe KW, Cartee GD, Weindruch R, Prolla TA (2008b) A low dose of dietary resveratrol partially mimics caloric restriction and retards aging parameters in mice. PLoS One 3(6):e2264
Beck MA (2007) Selenium and vitamin E status: impact on viral pathogenicity. J Nutr 137(5):1338-1340

Brenneisen P, Steinbrenner H, Sies H (2005) Selenium, oxidative stress, and health aspects. Mol Aspects Med 26(4-5):256267

Burk RF, Norsworthy BK, Hill KE, Motley AK, Byrne DW (2006) Effects of chemical form of selenium on plasma biomarkers in a high-dose human supplementation trial. Cancer Epidemiol Biomarkers Prev 15(4):804-810

Clark LC, Combs GF Jr, Turnbull BW, Slate EH, Chalker DK, Chow J, Davis LS, Glover RA, Graham GF, Gross EG, Krongrad A, Lesher JL Jr, Park HK, Sanders BB Jr, Smith CL, Taylor JR (1996) Effects of selenium supplementation for cancer prevention in patients with carcinoma of the skin. A randomized controlled trial. Nutritional Prevention of Cancer Study Group. JAMA 276(24):1957-1963

Connolly CD, Power RF, Hynes MJ (2003) Determination of selenium in animal feed by hydride generation and atomicabsorption spectroscopy: validation of analytical method. At Spectrosc 24(4):115-117

Duffield-Lillico AJ, Dalkin BL, Reid ME, Turnbull BW, Slate EH, Jacobs ET, Marshall JR, Clark LC (2003) Selenium supplementation, baseline plasma selenium status and incidence of prostate cancer: an analysis of the complete treatment period of the nutritional prevention of cancer trial. BJU Int 91(7):608-612

Giudice A, Arra C, Turco MC (2010) Review of molecular mechanisms involved in the activation of the Nrf2-ARE signaling pathway by chemopreventive agents. Methods Mol Biol 647:37-74

Hirota K, Matsui M, Iwata S, Nishiyama A, Mori K, Yodoi J (1997) AP-1 transcriptional activity is regulated by a direct association between thioredoxin and Ref-1. Proc Natl Acad Sci USA 94(8):3633-3638

Hoffman B, Liebermann DA (2009) Gadd45 modulation of intrinsic and extrinsic stress responses in myeloid cells. J Cell Physiol 218(1):26-31

Ip C, Birringer M, Block E, Kotrebai M, Tyson JF, Uden PC, Lisk DJ (2000) Chemical speciation influences comparative activity of selenium-enriched garlic and yeast in mammary cancer prevention. J Agric Food Chem 48(6):2062-2070

Ip C, Dong Y, Ganther HE (2002) New concepts in selenium chemoprevention. Cancer Metastasis Rev 21(3-4):281-289

Kabe Y, Ando K, Hirao S, Yoshida M, Handa H (2005) Redox regulation of NF-kappaB activation: distinct redox regulation between the cytoplasm and the nucleus. Antioxid Redox Signal 7(3-4):395-403

Keizer PL, Burgering BM, Dansen TB (2011) Forkhead Box O as a sensor, mediator, and regulator of redox signaling. Antioxid Redox Signal 14(6):1093-1106

Kim SY, Volsky DJ (2005) PAGE: parametric analysis of gene set enrichment. BMC Bioinf 6:144

Kipp A, Banning A, van Schothorst EM, Méplan C, Schomburg L, Evelo C, Coort S, Gaj S, Keijer J, Hesketh J, Brigelius-Flohé R (2009) Four selenoproteins, protein biosynthesis, and Wnt signalling are particularly sensitive to limited selenium intake in mouse colon. Mol Nutr Food Res 53(12):1561-1572

Koh TS, Benson TH (1983) Critical re-appraisal of fluorometric method for determination of selenium in biological materials. J Assoc Off Anal Chem 66(4):918-926

Kowalska E, Narod SA, Huzarski T, Zajaczek S, Huzarska J, Gorski B, Lubinski J (2005) Increased rates of chromosome breakage in BRCA1 carriers are normalized by oral selenium supplementation. Cancer Epidemiol Biomarkers Prev 14(5):1302-1306

Lee CK, Klopp RG, Weindruch R, Prolla TA (1999) Gene expression profile of aging and its retardation by caloric restriction. Science 285(5432):1390-1393 
Lippman SM, Goodman PJ, Klein EA, Parnes HL, Thompson IM Jr, Kristal AR, Santella RM, Probstfield JL, Moinpour CM, Albanes D, Taylor PR, Minasian LM, Hoque A, Thomas SM, Crowley JJ, Gaziano JM, Stanford JL, Cook ED, Fleshner NE, Lieber MM, Walther PJ, Khuri FR, Karp DD, Schwartz GG, Ford LG, Coltman CA Jr (2005) Designing the selenium and vitamin E cancer prevention trial (SELECT). J Natl Cancer Inst 97(2):94-102

Lippman SM, Klein EA, Goodman PJ, Lucia MS, Thompson IM, Ford LG, Parnes HL, Minasian LM, Gaziano JM, Hartline JA, Parsons JK, Bearden JD III, Crawford ED, Goodman GE, Claudio J, Winquist E, Cook ED, Karp DD, Walther P, Lieber MM, Kristal AR, Darke AK, Arnold KB, Ganz PA, Santella RM, Albanes D, Taylor PR, Probstfield JL, Jagpal TJ, Crowley JJ, Meyskens FL Jr, Baker LH, Coltman CA Jr (2009) Effect of selenium and vitamin $\mathrm{E}$ on risk of prostate cancer and other cancers: the selenium and vitamin $\mathrm{E}$ cancer prevention trial (SELECT). JAMA 301(1):39-51

Liu B, Suyeoka G, Papa S, Franzoso G, Neufeld AH (2009) Growth arrest and DNA damage protein $45 \mathrm{~b}(\mathrm{x} 45 \mathrm{~b})$ protects retinal ganglion cells from injuries. Neurobiol Dis 33(1):104-110

Lovell MA, Xiong S, Lyubartseva G, Markesbery WR (2009) Organoselenium (Sel-Plex diet) decreases amyloid burden and RNA and DNA oxidative damage in APP/PS1 mice. Free Radic Biol Med 46(11):1527-1533

Mahmoud KZ, Edens FW (2003) Influence of selenium sources on age-related and mild heat stress-related changes of blood and liver glutathione redox cycle in broiler chickens (Gallus domesticus). Comp Biochem Physiol B Biochem Mol Biol 136(4):921-934

McSheehy S, Yang L, Sturgeon R, Mester Z (2005) Determination of methionine and selenomethionine in selenium-enriched yeast by species-specific isotope dilution with liquid chromatographymass spectrometry and inductively coupled plasma mass spectrometry detection. Anal Chem 77(1):344-349

Qin S, Gao J, Huang K (2007) Effects of different selenium sources on tissue selenium concentrations, blood GSH-Px activities and plasma interleukin levels in finishing lambs. Biol Trace Elem Res 116(1):91-102

Qin S, Huang K, Gao J, Huang D, Cai T, Pan C (2009) Comparison of glutathione peroxidase 1 and iodothyronine deiodinase $1 \mathrm{mRNA}$ expression in murine liver after feeding selenite or selenized yeast. J Trace Elem Med Biol 23(1):29-35

Rao L, Puschner B, Prolla TA (2001) Gene expression profiling of low selenium status in the mouse intestine: transcriptional activation of genes linked to DNA damage, cell cycle control and oxidative stress. J Nutr 131(12):3175-3181

Rayman MP (2005) Selenium in cancer prevention: a review of the evidence and mechanism of action. Proc Nutr Soc 64(4):527542

Reid ME, Duffield-Lillico AJ, Sunga A, Fakih M, Alberts DS, Marshall JR (2006) Selenium supplementation and colorectal adenomas: an analysis of the nutritional prevention of cancer trial. Int J Cancer 118(7):1777-1781

Seo YR, Kelley MR, Smith ML (2002a) Selenomethionine regulation of p53 by a ref1-dependent redox mechanism. Proc Natl Acad Sci USA 99(22): 14548-14553

Seo YR, Sweeney C, Smith ML (2002b) Selenomethionine induction of DNA repair response in human fibroblasts. Oncogene 21(23):3663-3669

Waters DJ, Shen S, Cooley DM, Bostwick DG, Qian J, Combs GF Jr, Glickman LT, Oteham C, Schlittler D, Morris JS (2003) Effects of dietary selenium supplementation on DNA damage and apoptosis in canine prostate. J Natl Cancer Inst 95(3):237-241

Waters DJ, Shen S, Glickman LT, Cooley DM, Bostwick DG, Qian J, Combs GF Jr, Morris JS (2005) Prostate cancer risk and DNA damage: translational significance of selenium supplementation in a canine model. Carcinogenesis 26(7):1256-1262

Rider SA, Davies SJ, Jha AN, Clough R, Sweetman JW (2010) Bioavailability of co-supplemented organic and inorganic zinc and selenium sources in a white fishmeal-based rainbow trout (Oncorhynchus mykiss) diet. J Anim Physiol Anim Nutr (Berl) 94(1):99-110

Wang ZG, Pan XJ, Zhang WQ, Peng ZQ, Zhao RQ, Zhou GH (2010) Methionine and selenium yeast supplementation of the maternal diets affects antioxidant activity of breeding eggs. Poult Sci 89(5):931-937

Zainal HA, Wold WR, Water RM (1998) An NMR spectroscopic investigation of the oxidation reactions of DL-selenomethionine. J Chem Technol Biotechnol 72(1):38-44 